\title{
RESOLUCIÓN DE PROBLEMAS CIENTÍFICOS DESDE LA HISTORIA DE LA CIENCIA: RETOS Y DESAFÍOS PARA PROMOVER COMPETENCIAS COGNITIVO LINGÜÍSTICAS EN LA QUÍMICA ESCOLAR*
}

\author{
History of Science in the resolution of scientific problems: \\ contributions to promote linguistic cognitive strengths \\ for school Chemistry
}

Johanna Patricia Camacho González ${ }^{1}$

Mario Quintanilla Gatica ${ }^{2}$

\begin{abstract}
Resumen: En este artículo proponemos abordar el enfrentamiento a la resolución de problemas en la química escolar por parte de los y las estudiantes asumiendo situaciones a las que se han enfrentado los científicos y las científicas en determinados y contextualizados momentos de la Historia de la Ciencia. Para ello, argumentamos algunos aspectos desde la literatura especializada en Didáctica de las Ciencias, que permiten sustentar esta propuesta. A continuación, describimos un episodio histórico sobre la discusión del S. XVIII entre Lavoisier y Priestley respecto a la descomposición del aire para ser utilizada como estrategia que promueva el desarrollo de las competencias cognitivolingǘsticas. Finalmente, planteamos algunas directrices para orientar al profesorado de química en el logro de estos propósitos.
\end{abstract}

Palabras clave: Resolución de problemas. Historia de la Ciencia. Competencias cognitivolingüísticas. Bomba de vacío.

\begin{abstract}
The objective of this article is to approach the resolution of problems in the construction of knowledge in school science. In first place, show some aspects from specialized Literature. Later, we describe a historical episode of the discussion of Lavoisier and Priestley in S.XVIII about the combustion of the air. This is to be used as a strategy that promotes linguistic cognitive strengths. Finally, we propose some methodological directives to orient professors of chemistry to profit from these findings.
\end{abstract}

Key words: Resolutions of problems. History of science. Linguistic cognitive strengths. Pump of emptiness.

\footnotetext{
*Elaborado a partir de versión preliminar presentada en la IX Conferencia Internacional de Historia y Filosofía de la Ciencia (Calgary -Canadá).

${ }^{1}$ Grupo de Reflexión en Enseñanza de las Ciencias e Investigación Didáctica Aplicada (GRECIA), Facultad de Educación, Pontificia Universidad Católica de Chile (PUC); <jpcamach@uc.cl>.

${ }^{2}$ Doctor en Didáctica de las Ciencias Experimentales y las Matemáticas; docente, Facultad de Educación Pontificia Universidad Católica de Chile (PUC); Grupo de Reflexión en Enseñanza de las Ciencias e Investigación Didáctica Aplicada (GRECIA). <mquintag@uc.cl>.

${ }^{1}$ Pontificia Universidad Católica de Chile, Facultad de Educación, Doctorado en Ciencias de la Educación Av. Vicuña Mackenna 4860, Campus San Joaquín Santiago de Chile 


\section{Introducción}

En la actualidad se reconoce que existe poca coherencia entre la enseñanza de la ciencia y los retos que se plantean en los diferentes contextos de la sociedad, sí bien se espera que los ciudadanos y las ciudadanas que participan en los procesos de educación científica contribuyan de manera autónoma, crítica y participativa en las decisiones de su vida cotidiana, se evidencia que la enseñanza de las ciencias en la escuela poco promueve estas finalidades. ¿A qué se debe esto?, según el aporte de diferentes investigaciones en el campo de la Didáctica de la Ciencias, se evidencia la reducida coherencia que existe entre la enseñanza de las ciencias en el aula y una alfabetización científica que permita a los y las estudiantes pensar e interpretar el mundo con teoría científica. La gran preocupación de los profesores y las profesoras de ciencias como lo señala Duschl (1997), es la falta de tiempo para tratar contenidos, los cuales no son mediados a través de procesos reflexivos y se presentan de manera aislada a las necesidades de los estudiantes, sin establecer relaciones significativas con la dinámica propia de las comunidades científicas y por tanto, los aprendizajes no son efectivos ni promueven una formación científica para la vida.

En los últimos años, se han sugerido diferentes iniciativas de innovación basadas en la investigación en didáctica de las ciencias naturales, que promueven cambios curriculares en la enseñanza de las ciencias, para instaurar el desarrollo de habilidades y destrezas que permitan a los estudiantes mediante procesos reflexivos la construcción y resignificación de los conocimientos científicos que aprenden. Estas habilidades y destrezas se han establecido en un campo teórico bastante complejo, dinámico y a la vez difuso denominado competencias. De acuerdo con lo que plantea uno de nosotros (QUINTANILLA, 2006), estas competencias, especialmente en el campo de la educación científica, se corresponden con la capacidad de las personas para afrontar situaciones nuevas a partir de los conocimientos aprendidos; lo que se contempla tanto en el ámbito cognitivo, valórico y cultural y, tal como lo señala Labarrere (2006), dependen del sujeto y de su contexto.

Entonces ¿Cómo contribuir a que la enseñanza de la ciencia en general y de la Química en particular desarrolle sujetos competentes, ciudadanos críticos y ciudadanas críticas que comprendan y utilicen efectivamente el conocimiento científico? En este artículo pretendemos contribuir a esta discusión desde un campo disciplinar como lo es la Historia de la Ciencia, entendida como una metaciencia que aporta a la Didáctica de las Ciencias y que a través de una postura epistemológica racionalista moderada entre filogenia y ontogenia puede dar cuenta de la construcción de conocimiento científico desde una visión naturalista pragmática, promoviendo la enseñanza de contenidos científicos a través de procesos de modelización donde se integran el pensamiento, el lenguaje y la acción, contribuyendo a una imagen de ciencia menos triunfalista, más humana y más prospera a la actividad cultural y política de las comunidades científicas (GIERE, 1992). En este artículo presentamos estas ideas a través de un episodio histórico $\mathbf{L a}$ bomba de vacío ¿Quién dijo qué, a propósito de qué? Este episodio 'paradigmático' en la historia de la Química, permite evidenciar que la construcción de conocimiento químico es problemática, surge en momentos y contextos determinados y que para que sea posible que evolucionen los conceptos científicos, los químicos hacen uso de sus capacidades cognitivo lingüísticas al sustentar sus propuestas de manera racional y razonable tal y como lo plantean los realistas pragmáticos (IZQUIERDO, 2000a; GIERE, 1992; TOULMIN, 1977). 
Resolución de problemas científicos desde la Historia ...

\section{Crisis en la enseñanza de la Química}

La incorporación de la Química en los niveles escolares de enseñanza básica y media en América Latina tiene la finalidad de proporcionar a los futuros ciudadanos adultos y las futuras ciudadanas adultas las herramientas básicas, para que sean capaces de entender la realidad que les rodea y puedan comprender el papel de la ciencia en nuestra sociedad y contribuir de alguna manera a transformarla. Como afirma Campanario (1999), este primer contacto con la ciencia debería contribuir a que los y las estudiantes desarrollen ideas adecuadas sobre la ciencia y el conocimiento científico y a que apliquen hábitos propios del pensamiento y razonamiento de la ciencia en su vida cotidiana.

No obstante, en la enseñanza de la Química como lo señala Izquierdo (2004), se ha evidenciado en los últimos años una crisis, que se manifiesta en las opiniones desfavorables de quienes, ya mayores, recuerdan la Química como algo incomprensible y aborrecible; en la falta sostenida de alumnos que desean tener cursos optativos de esta área científica y en la disminución sistemática y ascendente de estudiantes que escogen la Química como carrera profesional, particularmente en el área docente. Se establecen entre otras dos posibles causas de esta crisis, especialmente la enseñanza desde una perspectiva demasiado dogmática, alejada de las finalidades y valores de los estudiantes y en segundo lugar, que quizás la enseñanza de la Química se ha visto sólo desde la perspectiva de la enseñanza de ideas teóricas sin explicar suficientemente a qué tipo de intervención se refieren, por lo que la práctica se convierte para los alumnos en un ejercicio irracional conectando conocimientos que no son comprendidos ni útiles para ellos. Esta crisis, también queda en evidencia en las afirmaciones de los profesores y las profesoras de Química, quienes han señalado que la enseñanza de esta ciencia se caracteriza por la dificultad en aplicar los conocimientos y que usualmente los estudiantes creen que no les sirve para explicar el mundo en el que viven.

\section{Problematizar la enseñanza-aprendizaje de la Química desde una visión naturalizada}

¿Qué hacer para cambiar esto? Desde los planteamientos de Toulmin (1977), se señala que las disciplinas científicas van evolucionando en la medida que los científicos a través de sus ideales explicativos, que no representan sólo las esperanzas lógicamente coherentes de los científicos, sino también sus expectativas razonables sobre la disciplina y las posibilidades reales de la investigación científica, se enfrentan a los problemas de su quehacer cotidiano. De la misma manera, sí se concibe la visión naturalista pragmática que cita Giere (1992), se establece además la relación que existe entre la construcción cognitiva de los conocimientos científicos y la actividad cognitiva del propio sujeto que aprende. Este aspecto es bastante relevante sí se desea promover la capacidad metacognitiva en los y las estudiantes a través del enfrentamiento a la resolución de problemas como actividad científica seria y responsable, es decir, como lo señala Izquierdo (2005), la actividad de los químicos y de la Química escolar a través de la cual se construye conocimiento. Por ello, la resolución de problemas se ha concebido como una competencia básica que ha de ser desarrollada sistemáticamente en los planes de estudio de manera intencionada didácticamente (COUSO e LÓPEZ, 2005). 
Considerando lo anterior, la enseñanza de las ciencias debe plantear problemas para aprender ciencias, los cuales deben caracterizarse por ser problematizadores, auténticos y similares a situaciones que se desarrollan en los contextos científicos reales; pero también significativos para los estudiantes que aprenden; relevantes para la disciplina científica que se enseña; que promuevan los procesos reflexivos y que sean factibles de ser enfrentados por los estudiantes de manera similar a como han sido abordados por los químicos en la Historia de la Ciencia, a través de procesos de modelización científica donde se conjugan, mediante una dialéctica heurística, el problema presentado a los sujetos con el modelo teórico, haciendo emergente las hipótesis que permiten abordar la nueva situación de aprendizaje.

Aquí es importante señalar, que estas situaciones cientificas escolares problematizadoras (SCEP) que se propongan a los estudiantes deberán además considerar la creatividad como un proceso de desarrollo potente e intencionado, donde ésta juega un papel central en la generación de significados, que corresponderán a la acción contextual, que impone el reconocimiento de que este desarrollo constituye un fenómeno de naturaleza cultural (LABARRERE, 1997).

La resolución de problemas vista como una competencia científica, debe contemplarse desde los planos de análisis y desarrollo (LABARRERE e QUINTANILLA, 2002), a partir de los cuales el estudiante puede enfrentarse a la SCEP en diferentes niveles: a) el plano instrumental-operativo, que implican los recursos del sujeto o del grupo que resuelve la situación centrados en aspectos tales como el contenido, las relaciones que lo caracterizan, las soluciones posible y las estrategias, procedimientos, entre otros, de la misma manera en que los químicos hacen uso de los instrumentos y aparatos científicos que les permite dar cuenta de los fenómenos estudiados; b) el plano personal significativo, al considerar los procesos y estados personales de quien resuelve el problema ya que son de gran relevancia en la manera en cómo el sujeto se enfrenta a la situación de aprendizaje. Como se ha mencionado anteriormente, la ciencia es una actividad cognitiva y por ello, se asume que esta debe ser significativa no sólo en función de la teoría en sí misma, sino también para el sujeto. Y, finalmente c) el plano relacional social (o cultural), que es el espacio generado en la solución grupal, en la interacción colectiva y colaborativa entre los sujetos a través de procesos comunicativos y el conocimiento, sus representaciones y la conciencia que se tiene de éstos por parte de los sujetos. Así se demuestra que la actividad científica escolar (ACE), se desenvuelve en determinados ámbitos y que es posible, gracias a la participación colectiva de hombre y mujeres tal como lo ha evidenciado la propia Historia de la Ciencia.

Mediante este proceso de construcción de conocimiento científico tanto en el ámbito de los científicos (químicos) como el de los que aprenden ciencia (escolares), se van identificando, caracterizando y promoviendo determinadas competencias cognitivolingüísticas como las de: definición, explicación, argumentación y justificación, entre otras. Estas competencias son posibles si se vinculan con la teoría científica que permiten articular el pensamiento, el lenguaje y la acción en los sujetos, para dar cuenta de los nuevos conocimientos científicos construidos paulatinamente por ellos. Consideramos que a partir del estudio de diferentes episodios históricos que han sido relevantes para la evolución de la Química en el desarrollo de la humanidad con una intención didáctica específica, se puede promover el enfrentamiento a SCEP como actividades científicas escolares que permita la construcción del conocimiento y el aprendizaje de la Química, de tal modo que los estudiantes se vayan "apropiando de los lenguajes de las ciencias que constituyen la cultura científica, construidos a lo largo de los siglos [...] aprender el lenguaje científico es pensar, hablar, escribir y leer" (SANMARTÍ e IZQUIERDO, 1998, p. 181). 
Resolución de problemas científicos desde la Historia ...

\section{La Historia de la Química y el enfrentamiento a la resolución de problemas científicos escolares}

Antes de mencionar y argumentar los aportes de la Historia de la Ciencia a la resolución de problemas científicos en el aula, es importante situarse teóricamente para dar cuenta de qué concepto de Historia nos apropiamos en esta orientación. Como se ha hecho explicito, en los párrafos anteriores, asumimos la actividad científica desde una perspectiva profundamente humana, una construcción social que se desarrolla y aplica en diversos ámbitos para producir transformaciones; no hay descubrimiento ni justificación de conocimiento de manera 'ahistórica'. Existen diferentes contextos que interactúan y se desarrollan entre sí para hacer posible la ciencia como actividad humana (ECHEVERRÍA, 1995). Tales conocimientos se desarrollan y evolucionan en momentos particulares, situaciones específicas que históricamente se transforman según las necesidades de las instituciones, las personas, los ámbitos sociales, políticos, valóricos y culturales. Desde esta posición naturalista pragmática, la Historia de la Ciencia como metaciencia, según la analogía que propone Barona (1994, p. 41), corresponde a una "imagen cartográfica de la ciencia, un mapa físico o terrestre, en el que los objetos representados sólo alcanzan sentido en relación con los otros objetos que forman parte del mapa", en consecuencia los datos no tienen significado de manera aislada en las comunidades científicas, sino entre ellos y las teorías que existen en función de las concepciones y finalidades del momento, las que permiten o no su desarrollo y consolidación (LAKATOS, 1983; TOULMIN, 1977).

Así, la Historia de la Ciencia concebida desde una visión diacrónica considera el contexto social, político, valórico y cultural a partir de los cuales se desarrollaron dichos conocimientos científicos, como señala Kragh (apud IZQUIERDO et al., 2006) se considera ideal, en vista que como sujetos históricos que somos no es posible liberarnos de los valores de nuestro tiempo ni evitar completamente el empleo de patrones contemporáneos en su análisis. Debido a esto, se hace necesaria la elaboración de reconstrucciones históricas que sin haberse dado en el pasado, son interpretaciones serias, rigurosas e intencionadas y que pueden utilizarse como aporte para la enseñanza de la ciencia y la promoción de competencias cognitivolingüísticas, debido a que corresponden a situaciones que se desarrollaron en contextos científicos reales y que se enmarcan en contextos específicos y en el ser, saber, saber hacer y querer hacer de los químicos como lo señalan entre otros Quintanilla (2006), Lirés (2006) y Labarrere (2006), dimensiones que se relacionan profundamente con las competencias y que permite que el estudiante transite en uno u otro o de uno a otro plano de análisis y desarrollo.

Según aportes de otros investigadores (QUINTANILLA, IZQUIERDO e ADÚRIZ-BRAVO, 2005; SOLBES e TRAVER, 2001; IZQUIERDO, 2000b; KRAGH apud BARONA, 1994; MATHEWS, 1994), la enseñanza-aprendizaje de las ciencias toma nuevas perspectivas y enfoques como el desarrollo de competencias cognitivolingüísticas a través del enfrentamiento a la resolución de problemas científicos como un proceso de construcción de conocimiento científico escolar. Por ejemplo, al argumentar porque una teoría científica se desarrolló con más éxito que otra; qué hizo posible que evolucionará dicho conocimiento; cómo era el contexto en el qué se desarrolló y cómo influyó ese nuevo conocimiento en dicho momento; cómo se enfrentaban los químicos a los problemas teóricos, experimentales y profesionales; cómo argumentaban sus ideas científicas; qué instrumentos disponibles hicieron posible el desarrollo y consolidación de determinados conceptos; qué les permitieron concluir 
en su momento, son actividades de enseñanza-aprendizaje que pueden ser promovidas a través de los episodios históricos analizados en la clase de Química.

El reto que se propone para una nueva cultura de la Educación Científica, es abordar la enseñanza de la Química y la formación docente desde una perspectiva humana que permita que los estudiantes construyan conocimiento científico en las aulas, al enfrentarse a problemas científicos de manera similar a como lo hacen los químicos y las químicas (QUINTANILLA, IZQUIERDO e ADÚRIZ-BRAVO, 2005). Para ello, es un factor indiscutible la formación histórico epistemológica de los profesores y las profesoras de ciencias, porque sin ser historiadores deben conocer los orígenes de la ciencia que enseñan, ser autónomos para seleccionar las temáticas que consideran relevantes dentro de la disciplina, articularlas con otros contextos y saber eruditos que sean importantes para los alumnos, promover actividades problematizadoras que los estimulen para que construyan conocimientos científicos con las teorías que se proponen desde la Química.

Para considerar esta propuesta y según lo plantea García (2003), sí el objetivo es que a través de la resolución de problemas se construya conocimiento científico escolar, es indispensable que las situaciones que se van a contemplar cumplan al menos con los siguientes objetivos: a) ser capaces de crear motivos de estudio y b) constituirse en una dificultad cognoscitiva para el estudiante, pero factible de resolver. Las fuentes que se pueden emplear para la elaboración de situaciones con estas características según Garret (1989) y Cortés (1989) son: a) los procesos de resolución de problemas actuales, situaciones que en este momento sean relevantes tanto para el ámbito científico como para el social; b) los procesos pasados: qué hicieron los científicos, en otros tiempos, cuáles fueron sus intereses y problemas, cómo trataron de resolverlos y por qué, tendiendo en cuenta las precisiones mencionadas previamente, a propósito de cómo intencionar el episodio histórico y desde qué visión de Historia de la Ciencia y c) los procesos históricos: cómo han cambiado las ideas, preguntas y técnicas a lo largo de los años. Creemos que en este tipo de fuente un modelo que se puede considerar con bastante rigurosidad y coherencias teórica y epistemológica, es el modelo de Toulmin (1977) al que hemos aludido en otros trabajos y cuerpos de investigación (CAMACHO et al., 2007).

A partir de lo señalado anteriormente proponemos un episodio histórico que describe la 'discusión química' entre Lavoisier y Pristley en el siglo XVIII, acerca de la descomposición del aire. A través de este episodio se puede mostrar cómo son las dinámicas de los químicos, sus juicios; prácticas experimentales; instrumentos; argumentos; debates; conflictos; acuerdos y pactos metodológicos entre otros, aspectos centrales en el momento en que estos químicos se enfrentaron al problema de la combustión como una nueva explicación científica.

\section{El episodio: la bomba de vacío ¿Quién dijo qué, a propósito de qué?3}

En el Siglo XVII en Alemania, Otto von Guericke, durante una demostración que llevó a cabo en Magdeburgo, adaptó a un tonel de madera una bomba de agua, lo llenó de

${ }^{3}$ Episodio basado en Braga et al. (2000a), Braga et al. (2000b); y Brock (1992). 
agua y cerró. Luego con la ayuda de varios hombres procedió a sacar el agua y dado a que el bombeo se había prolongado después de vaciado el tonel, se ocasionó la precipitación del aire a través de los poros de la madera. Este experimento motivó a Guericke a uno nuevo: la fabricación de una esfera de cobre a la que se le podía colocar una bomba, esta vez omitió el agua y bombeó directamente el aire. Cuando había extraído aparentemente todo el aire, la esfera se deformó de manera repentina (sufrió un efecto de compresión) debido a la presión atmosférica, así variando las condiciones iba creando nuevas situaciones que le permitían dar cuenta del vacío.

En 1654, de manera un tanto teatral, hizo la presentación de un nuevo experimento llamado: "los hemisferios de Magdeburgo", este consistió en unir un par de semiesferas unidas y dentro de ellas se hacía el vacío. La esfera así formada era separada con gran dificultad por un equipo de ocho caballos en cada lado (Figura 1).

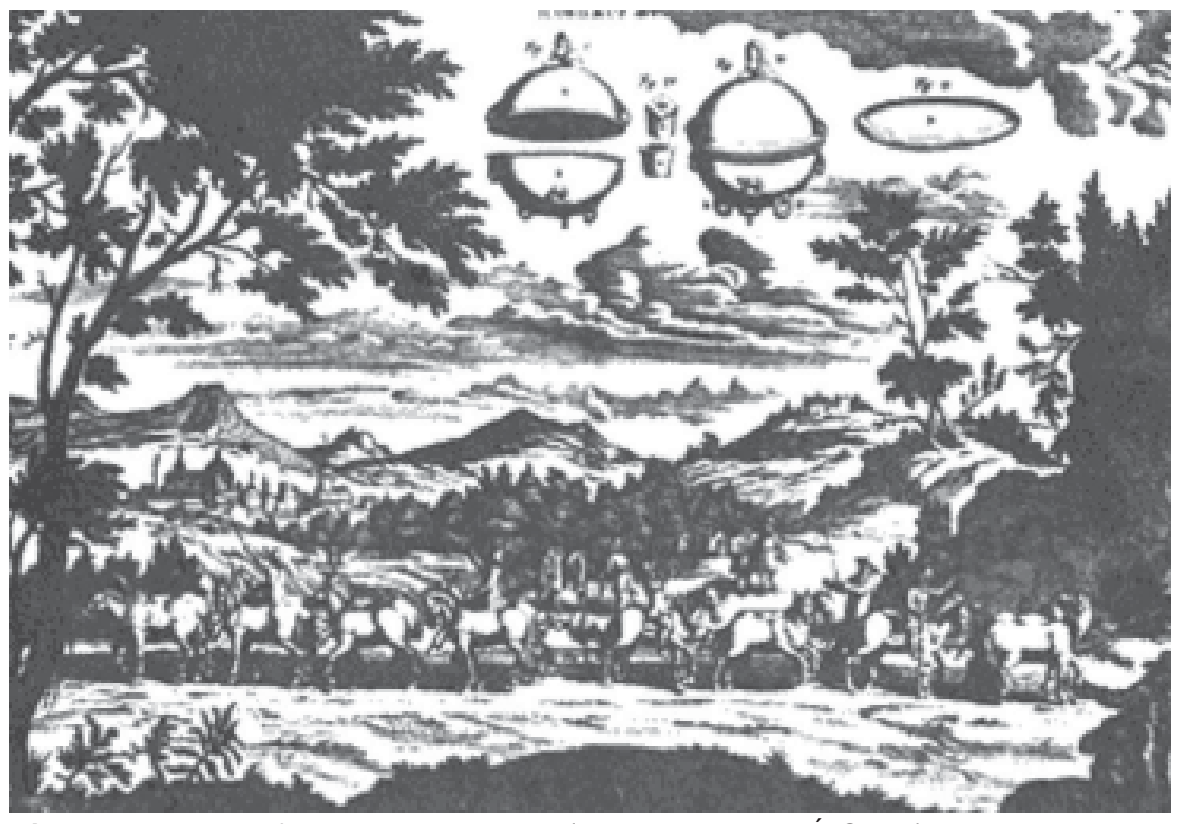

Figura 1. Los hemisferios de Magdeburgo (TALAVERA e FARÍAS, s.d).

Este tipo de artefactos construidos por von Guericke, fueron denominados en ese momento como jeringas, años después Robert Boyle quien también se interesó por el estudio del vacío, retomo este aparato científico durante sus estudios experimentales en 1658 y le nombró como bomba de vacío. 
Boyle, químico británico, junto con su ayudante Robert Hooke estuvieron interesados además en el estudio de la naturaleza de la combustión y de la respiración, cuyos experimentos fueron publicados en 1660 bajo el título de Nuevas experiencias físico-mecánicas tocando el espiritu del aire y sus efectos. Dado a la gran utilidad que le dio el equipo de Boyle a esta bomba, utilizando animales, plantas que le permitieron afirmar que el aire actuaba como un agente transportador, un fluido de elástico peculiar en el que flotaban las distintas partículas reactivas responsables de los fenómenos de respiración y la oxidación del hierro, los químicos de aquel entonces hablaban del vacio Boyleniano y estos trabajos se consideraron de gran importancia en los laboratorios de la Inglaterra del S. XVIII para continuar con investigaciones a partir de los resultados encontrados. Por ejemplo, los trabajos de Hooke que se caracterizaban por la creatividad que tenía para diseñar y construir instrumentos, algunos de ellos como el cuadrante, el anemómetro que permitían estudiar las condiciones climáticas de Inglaterra, durante su tiempo.

El también británico Joseph Black, utilizó la bomba de Boyle para estudiar algunos compuestos orgánicos que cuando eran sometidos a determinadas condiciones de calor desprendían un gas particular, al que Black le asigno el nombre de aire fijo, porque era característico de todas las sustancias y sólo sí las sustancias tenían presencia con ácidos, este se desprendía. Este aire al que se refería Black, años después se le dió el nombre de dióxido de carbono, el nombre con el que le conocemos en la actualidad.

A finales del siglo XVIII, los químicos tenían varias inquietudes sobre cómo se llevaban a cabo los cambios químicos y para ello, algunos decidieron estudiar la combustión de los cuerpos, entre ellos un joven químico británico, Joseph Priestley. Él se inscribía en las ideas que proponía Stahl y argumentaba sus observaciones desde la teoría del flogisto. En el año de 1774, realizó diversos experimentos, a través de los cuales pudo afirmar que el oxido rojo de mercurio obtenía un aire incoloro, de la misma manera que lo había mencionado Boyle años atrás, este gas nutría la llama de una vela. El aumento del fuego en presencia de ese aire, fue la característica por la que Priestley nombró a este gas como aire desflogisticado, dado a que la ausencia de flogisto en el aire se debía a que este salía con más intensidad del fuego para ocupar el espacio vacío existente en el aire. Esta conclusión, era sustentada además por los trabajos que desarrollaba dos años atrás el farmacéutico sueco Carl Scheele (contemporáneo de Lavoisier) que fueron conocidas sólo hasta 1777.

Desde otra posición teórica, que no aceptaba la idea del flogisto y por el contrario, buscaba nuevos conocimientos que dieran cuenta de ello. En Francia Lavoisier quien tuvo conocimientos de algunas de las conclusiones que se suscitaban en Inglaterra a través de los artículos escritos y divulgados en aquella época y que tenía especial interés por estudiar la posibilidad de experimentos sin la presencia de carbón. Lavoisier, sustentaba a través de evidencia empírica nuevas interpretaciones sobre el aire desflogisticado que era discutido por Priestley y que tenía características similares a la mencionada por Scheele. Según el francés, este era un gas que hacía parte del aire atmosférico de la misma manera que el gas fijo que mencionaba Black. Lavoisier consideraba a finales del S.XVIII que las conclusiones de los químicos ingleses eran equivocadas y por ello, planteo la necesidad de crear una nueva química.

Utilizando la tina neumática, Lavoisier, explicó los fenómenos de la combustión y la calcinación de metales y concluyó que el aire atmosférico no era un elemento puro, sino que constituía un conjunto de gases y estos participaban activamente en la combustión y en la calcinación. El aire incoloro que Priestley había identificado era una parte combustible del aire 
Resolución de problemas científicos desde la Historia ...

atmosférico y por ello, tornaba una llama en la vela de mayor intensidad. Como Lavoisier no compartía la teoría en la que Priestley se inscribía, decidió cambiar el nombre del aire desflogisticado por aire vital, que tras los años, tomo el nombre de oxígeno.

A partir del episodio histórico anterior, ¿qué actividades se pueden sugerir para promover el desarrollo de competencias cognitivolingüísticas en los estudiantes? ¿Cómo hacerlo? En el texto citado, se daba cuenta de la descomposición del aire, fenómeno que fue estudiado por varios científicos desde diferentes marcos teóricos según el conocimiento disponible en ese momento. Sí se considera este antecedente relevante para enseñar Química, se pueden identificar y caracterizar problemas que permitan que los estudiantes se enfrenten a esta situación de manera similar a como la abordaron los químicos en el siglo XVIII y que, años posteriores permitió que se establecieran nuevas preguntas que contribuyeron a consolidar las nuevas teorías de la Química.

\section{Una propuesta metodológica para enseñar a los estudiantes a enfrentarse a la resolución de problemas y promover competencias cognitivolingüísticas}

Hemos planteado en otras publicaciones e innovaciones con docentes en ejercicio, algunas directrices metodológicas para identificar, caracterizar y promover competencias científicas en el aula mediante el enfrentamiento a la resolución de problemas científicos específicos (QUINTANILLA et al., 2007; QUINTANILLA, 2006). Al respecto, indicamos previamente que el movimiento del sujeto que aprende por los planos de análisis o espacios de la resolución de un problema científico determinado, puede tener lugar en uno sólo de ellos o como tránsito de uno a otro; de manera que si, a partir de los fragmentos del discurso, o de la observación de la 'actividad científica' de resolución esta incluye entre otros, los criterios de análisis del problema, los modelos teóricos involucrados y los mecanismos y estrategias cognitivas que anticipan la acción de resolución y su sentido. Esto es muy importante, porque supone orientar la autorregulación de los procesos de aprendizaje científico y del control de los planos del desarrollo por parte del sujeto que aprende de manera intencionada para abordarlos y desarrollar competencias cientificas escolares (CCE).

Por ejemplo, en relación al tema histórico presentado (la bomba de vacío) sí un o una estudiante está tratando de establecer cuál es el 'contenido científico' de este 'contenido histórico', sí se puede referir o no a la bomba de vacío y sí es posible coordinarle determinado procedimiento de resolución, entonces él o ella se está moviendo en el plano instrumental operativo; en este caso, los objetivos que actúan se refieren a la comprensión de la estructura del problema histórico, el conjunto de relaciones que la singularizan, sus partes y los instrumentos de solución que pueden resultar viables según 'la densidad y coherencia' del modelo teórico que tenga acerca del contenido específico (bomba de vacío). Para promover competencias científicas a partir de un episodio histórico, hemos propuesto a profesores y profesoras en ejercicio, que participan de nuestra postura epistemológica, unas orientaciones o directrices teóricometodológicas que se pueden resumir en: a) Identificación de problemas científicos y commnicación de significados; b) problematizar e identificar tipologías de competencias científicas y c) evaluación de la experiencia con los estudiantes. A continuación se resumen brevemente las tres directrices señaladas. 


\section{Identificación de problemas científicos y comunicación de significados}

En este sentido, lo que interesa es identificar un 'problema científico' (concepto-ideapregunta problematizadora) en la historia de la ciencia; seleccionar la tipología o dimensión del problema incluido en el episodio histórico (conceptual, procedimental, actitudinal); identificar la teoría científica que subyace al problema seleccionado en la historia de la ciencia (¿Qué modelo teórico se quiere enseñar?); discriminar el nivel escolar en que se enseña ese contenido 'curricularmente' y transformarlo en una situación científica escolar problemática (SCEP) y contemplar la ideas previas (IP) de los alumnos/destinatarios. Finalmente identificar 'el plano de desarrollo' en que está formulado inicialmente el problema a los alumnos (instrumental, personal, comunicativo).

\section{Problematizar e identificar tipologías de competencias científicas}

En esta directriz se trata de vincular la SCEP con alguna competencia específica que se quiera desarrollar(ver Tabla 1); comunicar a los alumnos (o consensuar con ellos) el tipo de competencia cognitivolinguistica y sugerencias para enfrentarse a la resolución del problema que se ha enunciado y que está basado en el episodio histórico; enseñar a los alumnos a identificar el plano de análisis por donde se puede 'movilizar la SCEP'; identificar y disutir inicialmente con los estudiantes el marco teórico del problema científico, el marco procedimental y los recursos que posibilitan enfrentarse a resolver el problema (algorítmicos y heurísticos).

\section{Evaluación de la experiencia con los estudiantes}

En esta directriz se trata de potenciar la metacognición con los alumnos y alumnas con preguntas tales como ¿Qué reflexiones potenció el enfrentamiento al problema? ¿En qué planos del desarrollo lo situamos? ¿Por qué? ¿Cuáles fueron los criterios para evaluar el problema científico y cómo enfrentarlo inicialmente? ¿Cómo los identificamos? ¿Cuáles fueron las principales dificultades del análisis? ¿Cómo las identificamos y las superamos? ¿Qué competencias científicas desarrollamos o aprendimos? Es importante que el docente y los alumnos no pierdan de vista el modelo teórico que se ha seleccionado a partir del episodio histórico, ni tampoco la perspectiva de que hay 'buenas o malas' respuestas. Este es un momento privilegiado para 'mirar lo vivido' por el grupo de estudiantes y darse cuenta de cómo se está 'pensando teóricamente' con una finalidades humanas. Las directrices aquí presentadas se ejemplifican en la Tabla 1. 
Resolución de problemas científicos desde la Historia ...

Tabla 1. Directrices para promover competencias científicas cognitivolingüísticas a partir del episodio histórico seleccionado: La bomba de vacío ¿Quién dijo qué a propósito de qué?

\section{Identificación de problemas científicos y comunicación de significados}

1. Identificación de un "problema científico" (concepto-idea-pregunta problematizadora)

2. Seleccionar tipología o dimensión del problema

¿Cuáles son los componentes del aire?

\begin{tabular}{|l|l}
\hline Procedimental & $\begin{array}{l}\text { Uso de la bomba de vacío y otros } \\
\text { instrumentos científicos. }\end{array}$ \\
\hline Actitudinal & $\begin{array}{l}\text { Acuerdos, pactos metodológicos, } \\
\text { juicios, valores y discusiones de los } \\
\text { químicos. }\end{array}$
\end{tabular}

3. Identificar la teoría científica que subyace
(¿qué modelo teórico se quiere enseñar?) químicos.

Teoría de la combustión

\begin{tabular}{|c|c|c|}
\hline \multirow[t]{3}{*}{$\begin{array}{l}\text { 4. Identificar el plano de desarrollo en que } \\
\text { está formulado inicialmente el problema } \\
\text { científico desde la Historia de la Ciencia }\end{array}$} & $\begin{array}{l}\text { instrumental- } \\
\text { operativo }\end{array}$ & $\begin{array}{l}\text { - ¿Cómo crees tú que Priestley y } \\
\text { Lavoisier interpretaban el fenómeno de } \\
\text { la producción de un nuevo aire? } \\
\text { - ¿Qué criterios crees que utilizó } \\
\text { Priestley para hablar de aire } \\
\text { desflogisticado? } \\
\text { - ¿Qué prácticas experimentales } \\
\text { utilizarías para dar cuenta del "nuevo } \\
\text { aire" la bomba que utilizó Priestley o la } \\
\text { de Lavoisier, por qué? } \\
\text { - ¿Qué sucedió con lo que propusó } \\
\text { Scheele, tuvo alguna incidencia en la } \\
\text { discusión entre Lavoisier y Priestley, } \\
\text { qué crees y por qué? } \\
\text { - ¿Crees que sí Boyle, hubiese } \\
\text { trabajado en la misma situación, en la } \\
\text { misma época y con la bomba que } \\
\text { construyó, hubiera concluido algo similar } \\
\text { a lo de Priestley o Lavoisier? }\end{array}$ \\
\hline & $\begin{array}{l}\text { personal- } \\
\text { significativo }\end{array}$ & $\begin{array}{l}\text { - ¿Cómo crees que influyó el hecho } \\
\text { que Priestley era inglés y Lavoisier } \\
\text { francés? } \\
\text { ¿Cómo definirías el oxígeno y el aire } \\
\text { desflogisticado? } \\
\text { importante? }\end{array}$ \\
\hline & $\begin{array}{l}\text { relacional } \\
\text { social o } \\
\text { cultural }\end{array}$ & $\begin{array}{l}\text { - ¿Cómo relacionas el problema de la } \\
\text { descomposición del aire con situaciones } \\
\text { cotidianas? } \\
\text { - Discute con tus compañeros sobre la } \\
\text { actividad científica propuesta, cómo la } \\
\text { abordaste, cuáles fueron tus } \\
\text { debilidades y fortalezas. }\end{array}$ \\
\hline
\end{tabular}

continua 


\section{Problematizar e identificar tipologías de competencias}

1. Vincular el tipo de problema con alguna competencia específica que se quiera desarrollar
- ¿Cuáles fueron las ideas de Priestley y de Lavoisier acerca de la descomposición del aire? Describe - ¿Por qué Priestley habla de aire desflogisticado y Lavoisier de oxígeno? Justifica

- ¿Estás de acuerdo con las conclusiones de Priestley o de Lavoisier acerca de la descomposición del aire? Argumenta

2. Comunicar a los y las estudiantes el tipo de competencia científica y sugerencia para resolver el problema enunciado desflogisticado";
Argumenta a favor o en contra, las siguientes afirmaciones: - "Lavoisier vio oxígeno donde Priestley había visto aire

- "Los químicos ingleses estaban equivocados"; "no existía aire desflogisticado sino oxígeno" posiciona tus ideas desde lo que proponían en aquel entonces.

3. Enseñar a los y las estudiantes a identificar el plano de análisis en el que

4. Identificar con los y las estudiantes el marco teórico, procedimental y los recursos que posibilitan a enfrentarse a resolver el problema (algorítmicos y heurísticos) reflexionan el problema científico

\begin{tabular}{|c|c|}
\hline $\begin{array}{l}\text { instrumental- } \\
\text { operativo }\end{array}$ & $\begin{array}{l}\text { ¿Cuáles fueron los recursos } \\
\text { (conceptuales e instrumentales); los } \\
\text { procedimientos y las estrategias que } \\
\text { permitieron a Pristley y Lavoisier } \\
\text { estudiar el aire? }\end{array}$ \\
\hline $\begin{array}{c}\text { personal- } \\
\text { significativo }\end{array}$ & ¿Quiénes eran Pristley y Lavoisier? \\
\hline $\begin{array}{c}\text { relacional } \\
\text { social o cultural }\end{array}$ & $\begin{array}{l}\text { ¿Cómo fue la interacción colectiva y } \\
\text { colaborativa entre los químicos al dar } \\
\text { cuenta de la"nueva sustancia"? }\end{array}$ \\
\hline Marco teórico & $\begin{array}{l}\text { - Teoría del flogisto de Sthal } \\
\text { - Teoría de la combustión de Lavoisier } \\
\text { - Concepto de elemento de Boyle } \\
\text {. Estudio de los gases en el Siglo. XVIII }\end{array}$ \\
\hline $\begin{array}{c}\text { Marco } \\
\text { Procedimental }\end{array}$ & . Uso de la bomba de vacío \\
\hline $\begin{array}{l}\text { Recursos } \\
\text { Heurísticos }\end{array}$ & . "V" de Gowin \\
\hline
\end{tabular}

\section{Evaluación de la experiencia con los y las estudiantes}

1. ¿Qué reflexiones potenció el enfrentamiento al problema? ¿En qué planos de desarrollo lo situamos?

2. ¿Cuáles fueron los criterios para evaluar el problema científico y cómo enfrentarlo? ¿Cómo lo identificamos?

3. ¿Cuáles fueron las principales dificultades de análisis? ¿Cómo lo identificamos y superamos?

4. ¿Qué competencias científicas desarrollamos y aprendimos? 


\section{Reflexiones finales}

A partir de esta propuesta que hemos presentado, queda en evidencia la importancia que tiene la relación entre filogenia y ontogenia a lo largo de la Historia de la Ciencia para dar cuenta de la construcción de conocimiento científico a través de la resolución de problemas. La investigación en Didáctica de las Ciencias señala semejanzas entre la actividad cognitiva humana y la actividad científica individual y colectiva de las comunidades científicas que dan fundamento a los modelos de enseñanza constructivista (IZQUIERDO, 2000b). Por lo tanto, incorporar aspectos histórico epistemológicos en la enseñanza aprendizaje de la Química, con una aproximación intencionada didácticamente, no sólo proporcionará nuevas maneras de enseñar ciencias sino además nuevas concepciones de lo que es la actividad científica y de la importancia que esta tiene en diversos y variados contextos de la vida para asumir una posición crítica y antidogmática de esta actividad, aspectos centrales dentro de lo que contemplamos aquí como competencia científica escolar. Así, el uso de la Historia de la Ciencia permite que los alumnos y alumnas aprendan a enfrentarse a un problema científico real, sí este se presenta como problema para aprender a aprender, podrá promover de manera significativa competencias cognitivolingüísticas para la construcción y reelaboración conciente de conceptos y teorías científicas que permitan interpretar y transformar el mundo real.

La investigación, identificación, caracterización y selección de los diferentes episodios históricos para una clase de ciencias, como el que se presentó en este artículo, permiten a los y las estudiantes visualizar la dinámica progresiva de la comunidad científica, lo mismo que la manera como se formulan, argumentan, describen, justifican y debaten los problemas de los químicos; cuestiones que han de aprender a potenciar los y las estudiantes en la clase de Química. En este caso, estudiar el fenómeno de la combustión; la descomposición del aire; la existencia de nuevos gases; las características de las sustancias; justificar ideas por parte de los químicos y argumentar nuevas ideas en contraposición de las ya existentes. Dentro de este análisis se puede potenciar además el análisis, desarrollo y evolución de los conceptos científicos, a la manera como lo propone Toulmin (1977) la progresión de algunos, el abandono de otros y algunas variantes de significados científicos en la Historia de la Ciencia que permiten que evolucione o no determinados conceptos.

Pensamos que esta propuesta metacientífica tiene un promisorio futuro para la formación docente y la enseñanza aprendizaje de la Química escolar de mejor calidad en la escuela. Al respecto, hay mucho que crear y avanzar en este sentido desde la Didáctica de las Ciencias. 
Camacho González, J. P. ; Quintanilla Gatica, M.

\section{Referencias}

BARONA, J. Ciencia e Historia: debates y tendencias de la historiografía de la ciencia. Valencia: Seminari d'estudis sobre la ciência, 1994.

BRAGA, M et al. Breve história da ciência moderna: das luzes ao sonho do doutor Frankestein (séc. XVIII). Rio de Janeiro: Jorge Zahar, 2000a. v. 3.

Lavoisier e a ciência no ilumnismo. São Paulo: Atual Editora, 2000b.

BROCK, W. Historia de la Química. Madrid: Alianza Editorial, 1992.

CAMACHO, J.et al. La Ley Periódica analizada desde el modelo de Toulmin. Aportes para la enseñanza de la Historia de la Química. In: QUINTANILLA, M. (Ed.). Historia de la Ciencia: Propuestas para su divulgación y enseñanza. Chile: Arrayán, 2007. v. 2. p. 107-124.

CAMPANARIO, J. La ciencia que no enseñamos. Enseñanza de la Ciencias, v. 17, n. 3 , p. 397-410, 1999.

CORTÉS, L. The use of problem: solving in the history of chemistry course. Journal of Chemical Education, v. 69, n. 12, p. 1012-1013, 1989.

COUSO, D.; LÓPEZ, J. El problemes dels «problemes»: anàlisis i transformació de 1' enunciat de problemes de «paper i llapis». In: IZQUIERDO, M. (Ed.). Resoldre problemes per aprendre. Bellatera: Universitat Autònoma de Barcelona, 2005. p. 35-44.

DUSCHL, R. Renovar la enseñanza de las ciencias: importancia de las teorías y su desarrollo. España: Narcea, 1997.

ECHEVERRÍA, J. Filosofía de la Ciencia. Madrid: Akal Ediciones, 1995.

GARCÍA, J. J. Didáctica de las ciencias: resolución de problemas y desarrollo de la creatividad. Bogotá: Editorial Magisterio, 2003.

GARRET, R. Resolución de problemas, creatividad y originalidad. Revista Chilena de Educación Química, v. 14, n. 1-2, p. 224-230, 1989.

GIERE R. La explicación de la ciencia: un acercamiento cognitivo. México: Consejo Nacional de Ciencia y Tecnología, 1992.

IZQUIERDO, M. Com fer problemàtiques els problemes que no en són proa. Noves temàtiques per als problemes de química. In: (Ed.). Resoldre problemes per aprendre. Bellatera: Universitat Autònoma de Barcelona, 2005. p. 45-52.

Un nuevo enfoque de la enseñanza de la química: contextualizar y modelizar. The Journal of the Argentin Chemical Society, v. 92, n. 4/6, p. 115 -136, 2004.

Fundamentos epistemológicos. In: PERALES, F. J.; CAÑAL, P. (Eds.). Didáctica de las ciencias experimentales: teoría y práctica de la enseñanza de las ciencias. España: Marfil, 2000a. p. 35-64.

. Relaciones entre la Història i la didactica de les ciencias. In: TROBADA D'HISTÒRIA DE LA CIÈNCIA I DE LA TÈCNICA, 5., 2000, Barcelona. Actas... Barcelona: Societat Catalana d'història de la Ciència i de la Tècnica, 2000b. 
Resolución de problemas científicos desde la Historia ...

. et al. Relación entre la historia y la filosofía de las ciencias II. Alambique, n. 48, p. 78-91, 2006.

LABARRERE, A. De la competencia al sujeto competente y más allá. Una Historia en tres partes. In: SEMINARIO INTERNACIONAL DE DIDÁCTICA DE LAS CIENCIAS NATURALES. COMPETENCIAS CIENTÍFICAS, FORMACIÓN DOCENTE Y APRENDIZAJE PARA UNA CULTURA CIUDADANA, 7., 2006, Santiago. Anais... Santiago: Pontificia Universidad Católica de Chile, 2006.

Pensamiento: el análisis y la autorregulación de la actividad cognoscitiva de los alumnos - pueblo y educación. Pueblo y Educación: La Habana, 1997.

LABARRERE, A.; QUINTANILLA, M. La solución de problemas científicos en el aula. Reflexiones desde los planos de análisis y desarrollo. Pensamiento Educativo, v. 30, n. 1, p. 121-137, 2002.

LAKATOS, I. La metodología de los programas de investigación científica. Madrid: Alianza, 1983.

LIRÉS, M. Una aproximación a la enseñanza de las competencias científicas. In: SEMINARIO INTERNACIONAL DE DIDÁCTICA DE LAS CIENCIAS NATURALES. COMPETENCIAS CIENTÍFICAS, FORMACIÓN DOCENTE Y APRENDIZAJE PARA UNA CULTURA CIUDADANA, 7., 2006. Santiago. Anais... Santiago: Pontificia Universidad Católica de Chile, 2006.

MATHEWS, M. Historia, filosofía y enseñanza de las ciencias: la aproximación actual. Enseñanza de las Ciencias, v. 12, n. 2, p. 255-277, 1994.

QUINTANILLA, M. Identificación, caracterización y evaluación de competencias científicas desde una imagen naturalizada de la ciencia. In: QUINTANILLA, M.; ADÚRIZBRAVO, A. (Eds.). Enseñar ciencias en el nuevo milenio: retos y desafíos. Santiago: Pontificia Universidad Católica de Chile, 2006. p. 18-42.

et al. (Eds.). Estrategias innovadoras para el desarrollo de competencias cognitivolingüísticas mediante el enfrentamiento a la resolución de problemas. Santiago: Facultad de Educación, Pontificia Universidad Católica de Chile, 2007. [Material generado en el curso de actualización en didáctica de las ciencias naturales]

.; IZQUIERDO, M.; ADÚRIZ-BRAVO, A. Characteristics and methodological discussion about a theoretical model that introduces the history of science at an early stage of the experimental science teachers' professional formation. In: Science \& Education IHPST, 8., 2005, Leeds. On line... Disponível em: < http://www.ihpst2005.leeds.ac.uk/ papers/Quintinilla_Izquierdo_Aduriz-Bravo.pdf>. Acesso em: 10 dez 2006.

SANMARTÍ, N.; IZQUIERDO, M. Enseñar a leer y escribir textos en ciencias. In: JORBA, J.; GÓMEZ, L.; PRAT, A. (Eds.). Hablar y escribir para aprender. España: ICE de la UAB, 1998. p. 181-199. 


\section{Camacho González, J. P. ; Quintanilla Gatica, M.}

SOLBES, J.; TRAVER, M. Resultados obtenidos introduciendo historia de las ciencias en las clases de Física y Química: mejora de la imagen de la ciencia y desarrollo de actitudes positivas. Enseñanza de las Ciencias, v. 19, n. 1, p. 151-162, 2001.

TALAVERA, L.; FARÍAS, M. Los dispositivos para hacer vacío. In: E1 vacío y sus aplicaciones. Disponível em: <http://omega.ilce.edu.mx:3000/sites/ciencia/ volumen3/ciencia3/131/htm/sec_8.htm>. Acesso em: 10 dez. 2006.

TOULMIN, S. La comprensión humana: el uso colectivo y la evolución de conceptos. Madrid: Alianza Editorial, 1977. v. 1.

Artigo recebido em abril de 2007 e aprovado em junho de 2007. 